\title{
Neurodegeneration with brain iron accumulation
}

INSERM

\section{Source}

INSERM. (1999). Orphanet: an online rare disease and orphan drug data base.

Neurodegeneration with brain iron accumulation. ORPHA:385

Neurodegeneration with brain iron accumulation (NBIA, formerly Hallervorden-Spatz

syndrome) encompasses a group of rare neurodeg enerative disorders characterized by progressive extrapyramidal dysfunction (dystonia, rigidity, choreoathetosis), iron accumulation in the brain and the presence of axonal spheroids, usually limited to the central nervous system. 